\title{
Remote Video-to-Video Eye Telemonitoring Use Case for Glaucoma Patients
}

\author{
Dimitrios Alonistiotis $^{1}$, Evgenia Kontou ${ }^{1}$, Nikolaos Karachalios ${ }^{1}$, Eleni Patouni ${ }^{2}$, \\ Panagiotis Diamantopoulos ${ }^{2}$, Nikolaos Bompetsis ${ }^{2}$, Nancy Alonistioti ${ }^{2}$ \\ and Ioannis P. Chochliouros ${ }^{3}$ \\ ${ }^{1} 2^{\text {nd }}$ Department of Ophthalmology, University of Athens, \\ "Attikon" Hospital, \\ 1, Rimini Street, Haidari, GR-124 62, Athens, Greece \\ dalonis@yahoo.gr \\ ${ }^{2}$ Department of Informatics and Telecommunications \\ National and Kapodistrian University of Athens, \\ 6, Panepistimiopolis, Ilissia, GR-15784, Athens, Greece \\ \{elenip, panos_10d, nbompetsis, nancy\}@di.uoa.gr \\ ${ }^{3}$ Research Programs Section, Fixed \\ Hellenic Telecommunications Organization (OTE) S.A., \\ 99, Kifissias Avenue, GR-151 24, Athens, Greece \\ ichochliouros@oteresearch.gr
}

\begin{abstract}
Glaucoma is the second leading cause of blindness globally and the second most common cause of avoidable visual impairment. It also holds a record in noncompliance to therapy from the patients in up to $50 \%$ of the subjects treated with anti-glaucoma eye drops. LiveCity e-Health is a European research program, which aims to provide better treatment and follow up of glaucoma patients at their home, through telemonitoring with high definition video-to-video (v2v) communication from the University Hospital. Secondly, it aims to reduce the cost of health and improve the city environment by decreasing the number of visits to the Hospital. For this purpose, a software application has been developed; the latter is easy to use for elderly people at home, and capable of keeping the medical history and digital records of every patient in the Glaucoma Department. In addition, a specific web camera with snapshot ability of high quality photo of the eye has utilised. Two patients have been initially enrolled in the study and the preliminary results are so presented.
\end{abstract}

Keywords: e-Health, glaucoma, telemonitoring, video-to-video (v2v) communication.

\section{$1 \quad$ Introduction}

According to the World Health Organization (WHO) and all the available surveys, glaucoma is the second leading cause of blindness globally as well as in most regions of the world, following only cataract, and the third in the developed world, following 
Age-related Macular Degeneration and Diabetic Retinopathy ([1]-[3]). It is also considered to be the second most common cause of avoidable visual impairment [4].

Glaucoma is an optic neuropathy with certain features including an intraocular pressure that is too high for the continued health of the eye, which leads to ganglion cell death. Ophthalmology so far has focused in the management of glaucoma through the lowering of the intraocular pressure and the preservation of the remaining visual ability [5]. This is achieved primarily by eye drop instillation daily and only in few selected cases by surgery or laser. Normal intraocular pressure values vary from 10 to $21 \mathrm{mmHg}$, and most glaucoma patients tend to have more than 24 before diagnosis [6]. Regaining some of the impaired vision due to glaucoma is impossible.

At the same time, glaucoma treatment heavily reduces the quality of life of the patient. The European Glaucoma Society has insisted that glaucoma therapy must abide by a good quality of life for the patient, according to the stage of the disease, the patient's age, general health and even life expectancy and also his abilities and needs [7]. The main reason that Glaucoma influences the patient's attitude towards his therapy is that it stays an asymptomatic disease until the end-stage of the glaucomatic damage (when preservation of vision is impossible by then) and can only be detected through preventive examination of the intraocular pressure and the optic nerve. Secondly, chronic use of eye drops causes topical side effects like redness of the eye, discomfort, itching, conjunctivitis, blurry vision, even hyperpigmentation of the iris and enlargement of the eye lashes in the most common anti-glaucoma drug category. Even systemic side effects from long lasting anti-glaucoma therapy, including depression, respiratory and cardiac side effects and sexual impotency have been noticed. So, in most cases, a healthy otherwise and asymptomatic individual who goes for a check-up to the Ophthalmologist and is suddenly considered and treated as glaucomatic ends up with many different symptoms not from the disease itself but from the medications with an aim to maintain - and not restore- his vision. Last but not least, the cost of therapy for the entire lifetime becomes an economic burden for the citizen.

For all the above reasons Glaucoma holds a record in noncompliance to therapy from the patients. Several surveys have shown that glaucomatic patients do not take their medications correctly in a percentage up to 50\% [8]. Besides that, because of the nature of the disease, it is estimated that there is also a $50 \%$ of undiagnosed glaucomatic patients in the general population and that almost half of the people under anti glaucoma therapy may not need it after all. Moreover, because many glaucoma patients need more than one medication (eye drop) and in different daily frequency or time of instillation (once or twice by day), are geriatric patients or with specific difficulties with applying medication, (e.g. due to tremor), noncompliance to therapy increases according to the number of eye drops per day, number of medications, age, general mental condition ([9]-[10]).

Pharmacy companies have tried to improve this by producing more effective drugs, reducing the daily need for eye drops, introducing drugs with less side effects and preservative free single use blisters. Nevertheless, glaucoma patients need close follow up for their obedience to therapy and even training for the correct instillation of eye drops [11]. 
The rest of the paper is organised as follows: Section 2 analyses the LiveCity Project research in the area of e-health, focusing on the description of the telemedicine platform and software application for remote patients' telemonitoring. The telemonitoring use case for glaucoma patients is introduced in section 3. Finally conclusion remarks and directions for future research are drawn in section 4.

\section{The LiveCity e-Health Research for Smart Cities}

The LiveCity Project [12] is based on live high-definition interactive video communication easily available on user equipment. LiveCity e-Health for glaucoma is a part of this European program, which aims to improve the quality of life of chronic patients, reduce their need for transportation to the Hospital and facilitate communication with the doctor through a high definition video-to-video telemonitoring from the patient's home [13]. More benefits from this include reduction of morbidity, limiting of therapy's side effects, reduction of healthcare cost because of increased compliance to therapy, less car traffic in the cities, reduced greenhouse emissions, less fossil fuel consumption, less air pollution.

For the glaucoma patients in particular, the study involves the Hellenic Telecommunications Organization S.A. (OTE), the Informatics \& Telecommunications Department of the National and Kapodistrian University of Athens and the Glaucoma Department of the $2^{\text {nd }}$ Ophthalmological University Clinic of Athens in Attikon Hospital. The essential aim is to communicate with glaucomatic patients at their home, from the Hospital, both in regular appointments and in case of emergency. Regular appointments include monitoring of their compliance to therapy, eye drop instillation reminding, eye drop instillation training and display [14], common examination with a high definition video-to-video communication and even the use of camera snapshots. At the same time, the patient can call the Glaucoma Department in case of emergency for a 24/7 follow-up. This may include cases of a common but very impressive subconjuctival haemorrhage which needs no transportation to the hospital and can be easily diagnosed from the camera, any acute symptoms from the eyes or side effects from therapy, even postsurgical examination. Authorized doctors have also the ability to send communication request to a specific patient, if needed.

\subsection{LiveCity Telemedicine Platform}

The LiveCity Telemedicine platform supports high definition video streaming between an eye doctor and a patient over public Internet. In this end, two different end-user applications, the Doctor Plugin and the Patient Plugin, have been designed and developed to address the needs of the doctor and the patient respectively. The plugin allows the doctor to establish high definition quality video-to-video communication with a chosen patient. The core functionality of the application is provided by a simple graphical user interface. Specifically, the following functionality is supported: 
- Connect and Disconnect: The doctor can register the client software in the LiveCity network and obtain access to the LiveCity services by pressing the button Connect and filling-in his certified credentials. By pressing the button Disconnect the doctor unregisters his client from the LiveCity network.

- Call: This button allows the doctor to establish video-to-video communication with patients that are currently connected to the LiveCity network.

- Notifications: The notifications area is used to inform the doctor that a patient requires his attention. When a patient issues a call request the application shows the caller's name in the notifications area informing the doctor.

- Feedback: This button provides the doctor the ability to report to the developers issues arising during the application use. The doctor can issue a report for a bug or unexpected application behavior providing a short description of the problem.

- Adv. Settings: Provides a set of additional configurations that extend the overall flexibility of the application. Through these configurations the doctor can change the network parameters of the application, determine new video and audio devices and even define the video resolution.

During an active video session the doctor can manually adjust the settings of his own video resolution. Through the Adv. Settings the doctor can choose between four individual video profiles: platinum (1080p video streaming), gold (720p video streaming), silver (480p video streaming) and bronze (360p video streaming).

In addition, the doctor can adjust the volume, contrast, brightness and saturation of the remote video. This functionality is available by pressing the button Controls. Moreover the doctor may save a snapshot of the received video by pressing the button Screenshot. The image is stored in the LiveCity database and can be used later by the doctors to assist the patient examination. The doctor may change the resolution of the streamed video using the button Remote Video. Finally, the doctor can terminate the call session by pressing the button Hang up.

The LiveCity Telemedicine Patient Plugin is a simple and lightweight application used by the patients and it mainly targets the inexperienced users. It provides exactly the same high definition video-to-video communication profiles and the same core functionality of the Hospital side plugin. It should be noted however, that the Patient Plugin does not provide the same call functionality. By pressing the call button the patient application sends a notification to the doctor application. Thus, the doctors can initiate a call, so that they have the full control of the video communication establishment.

The patient's access to the $\mathrm{v} 2 \mathrm{v}$ service is realized through a fixed and low-cost xDSL connection. In addition, at the hospital side, a high quality and bandwidth vDSL line has been established with the network provider for ensuring high quality video delivery to the medical personnel. The upload bandwidth utilization of the patient and, as a consequence, the download utilization of the doctor, is nearly linear at $1.3 \mathrm{Mbps}$. On the other hand, the upload utilization of the doctor is gradually increasing up to $3 \mathrm{Mbps}$, due to the additional traffic generated by the doctor movement during examination. 


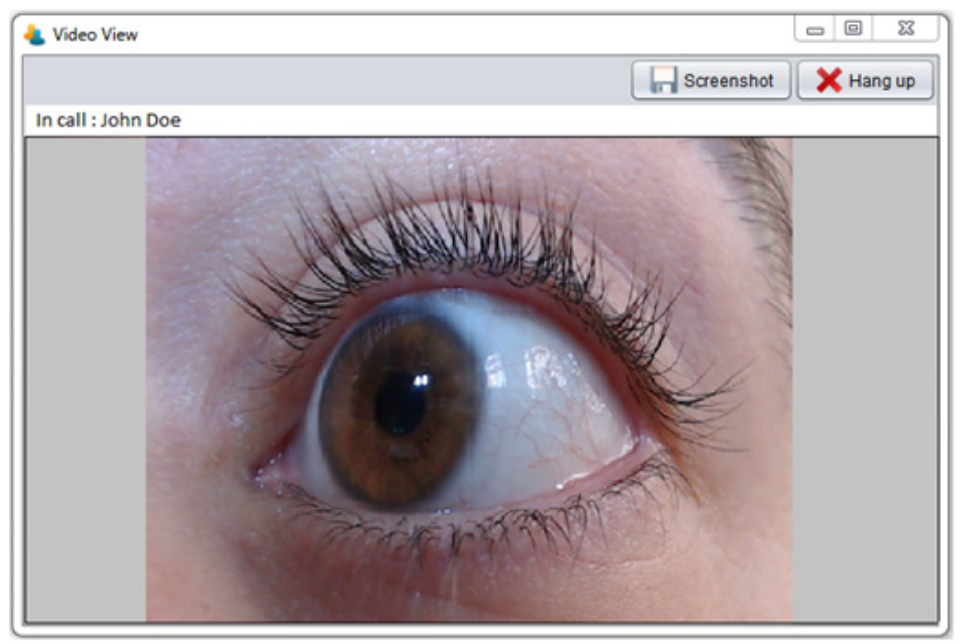

Fig. 1. Eye monitoring camera snapshot

Patients are equipped with common laptops supplied with the software developed for the LiveCity e-Health Project and with individual web cam. Camera snapshots have reached a quality of analysis better than the human eye and sometimes equal to that of an examination slit-lamp and can even detect a contact lens wearer (as shown on Fig.1).

\section{Telemonitoring Use Case for Glaucoma Patients}

The telemedicine plugin has been developed from the Informatics Department of the University of Athens and used from the Attikon Hospital in practise including: patient's medical history, baseline slit-lamp examination, intraocular pressure measurements, list of all available anti-glaucoma drugs, individual file for each patient with his previous appointment findings, comments and eye snapshots.

The technical part has absorbed most of the preparation time for this use case, allowing for only a short period for the patients' telemonitoring up to now. Training of the patients for the use of laptops, the web cam and the software is necessary, despite the fact that both the hardware and the software are user friendly. This is due to the geriatric patients [15] and their limited experience with the use of computers and Internet. Regular appointments are weekly and more often in the first month. The doctor-patient communication scenario for the eye telemonitoring is shown in Table 1.

For quality control we have decided to include in the study patients with borderline control of their glaucoma, with the purpose to improve the results of conservative therapy via increased compliance. Also, advanced stage patients are included in the study group with the aim to postpone surgical treatment through closer follow-up. Numerical measurements like initial and intermediate intraocular pressure values, number of drugs and the number of eye drops used for each eye are monitored 
throughout follow-up, in order to reach medical conclusions. Also, record is kept for the number of regular appointments, emergency calls and the number of visits that have been avoided because of video-to-video telemonitoring, for the final evaluation ([16], [17]). Subjective satisfaction report of each patient and doctor for the program and the quality of the video is asked. An unavoidable two (2) second delay in sound and video image has been noticed during the $\mathrm{v} 2 \mathrm{v}$ communication with the patients.

Table 1. Scenario of Doctor-Patient Communication in the LiveCity Project

\begin{tabular}{l|l|l}
\hline \multicolumn{1}{c|}{$\begin{array}{c}\text { Questions of Regular } \\
\text { Appointments }\end{array}$} & Questions of Emergency Call & $\begin{array}{l}\text { Additional Questions } \\
\text { for the First Call }\end{array}$ \\
\hline $\begin{array}{l}\text { General condition of the } \\
\text { treated eye. }\end{array}$ & What is the cause of the call? & $\begin{array}{l}\text { Ease of use of the } \\
\text { hardware/software. }\end{array}$ \\
\hline $\begin{array}{l}\text { Remark any troublesome } \\
\text { symptoms after starting } \\
\text { treatment }\end{array}$ & $\begin{array}{l}\text { When did the disturbing } \\
\text { symptoms appear? }\end{array}$ & $\begin{array}{l}\text { Level of satisfaction } \\
\text { with the new type of } \\
\text { communication. }\end{array}$ \\
\hline $\begin{array}{l}\text { Difficulty in applying } \\
\text { eye drops? }\end{array}$ & $\begin{array}{l}\text { What is the duration of the } \\
\text { symptoms? }\end{array}$ & \\
\hline $\begin{array}{l}\text { Demonstrate eye to the } \\
\text { camera. }\end{array}$ & $\begin{array}{l}\text { Is there any decrease in } \\
\text { vision? }\end{array}$ & \\
\hline $\begin{array}{l}\text { Application of eye drops } \\
\text { demo. }\end{array}$ & Feeling any pain? \\
\hline $\begin{array}{l}\text { Follow instructions for } \\
\text { implementing drip- } \\
\text { hours? }\end{array}$ & Demonstrate eye to camera. & \\
\hline $\begin{array}{l}\text { Confirm details for next } \\
\text { appointment. }\end{array}$ & $\begin{array}{l}\text { Possible questions from the } \\
\text { patient. }\end{array}$ & $\begin{array}{l}\text { Confirm details for next } \\
\text { appointment. }\end{array}$ \\
\hline $\begin{array}{l}\text { Possible questions from } \\
\text { patient. }\end{array}$ &
\end{tabular}

Because of the advanced technical infrastructure needed for the program and the cost for each patient included in this, so far only 2 patients (females, 70 and 81 years old respectively) have been enrolled and 2 more have been recruited and waiting for the connectivity establishment. A control group for comparison, with similar demographic characteristics but without access to LiveCity, has been formed. Because of the small number of participants so far, no comparative conclusions can be reached. Both the enrolled patients are scheduled for cataract extraction in the next 6 months and postoperative examination with video-to-video communication will be assessed (One patient is treated for glaucoma since 10 years with one medication - the second patient is newly diagnosed and under triple therapy).

\section{Conclusion}

Telemonitoring of glaucoma patients with high definition video-to-video communication is a novel approach. Various imaging examinations exist in everyday 
practice in Ophthalmology and the bases of the ophthalmological diagnosis. Glaucoma patients besides being suitable for tele-examination with high definition video and imaging have also a record in noncompliance to therapy. Telemonitoring is expected to improve training, reminding, correct instillation of eye drops and therefore compliance to therapy. Existing video platforms candidates for realizing v $2 \mathrm{v}$ services (e.g. Skype, Oovoo) do not offer any facility to integrate additional features to support such e-health use cases. The LiveCity Project develops a user-friendly software plugin that can be easily used in wide scale for community connection with the reference Hospital in smart cities [18]. A larger number of patients will be enrolled in the project in the next months for deriving more accurate statistical results.

Acknowledgments. The present article has been structured in the context of the LiveCity ("Live Video-to-Video Supporting Interactive City Infrastructure") European Research Project and has been supported by the Commission of the European Communities - DG CONNECT (FP7-ICT-PSP, Grant Agreement No.297291).

\section{References}

1. Resnikoff, S., Pascolini, D., Etya'ale, D., Kocur, I., Pararajasegaram, R., Pokharel, G.P., Mariotti, S.P.: Global Data on Visual Impairment in the Year 2002. Bulletin of the World Health Organization 82, 844-851 (2004)

2. World Health Organization (WHO): Prevention of Blindness and Deafness. Global Initiative for the Elimination of Avoidable Blindness. Geneva, WHO (2000). (WHO document WHO/PBL/97.61 Rev2)

3. World Health Organization: The World Health Report 2003: Shaping the Future. Geneva, WHO (2003)

4. Quigley, H.A., Broman, A.T.: The Number of People with Glaucoma Worldwide in 2010 and 2020. British Journal of Ophthalmology 90, 262-267 (2006)

5. Leske, M.C., Heijl, A., Hyman, L., Bengtsson, B., Komaroff, E.: Factors for Progression and Glaucoma Treatment The Early Manifest Glaucoma Trial. Current Opinion in Ophthalmology Journal 15, 102-106 (2004)

6. Heijl, A., Leske, M.C., Bengtsson, B., Hyman, L., Hussein, M.: Early Manifest Glaucoma Trial Group. Reduction of Intraocular Pressure and Factors for Progression and Glaucoma Progression: Results from the Early Manifest Glaucoma Trial. Archives of Ophthalmology 120, 1268-1279 (2002)

7. Kholdebarin, R., Campbell, R.J., Jin, Y.-P., Buys, Y.M.: Multicenter Study of Compliance and Drop Administration in Glaucoma. Canadian Journal of Ophthalmology 43(4), 454-461 (2008)

8. European Glaucoma Society: Terminology and Guidelines for Glaucoma, 3rd Edition. Savona, Editrice Dogma S.r.l (2008)

9. Sleath, B., Robin, A.L., Covert, D., Byrd, J.E., Tudor, G., Svarstad, B.: Patient-reported Behavior and Problems in Using Glaucoma Medications. Ophthalmology Journal 113, 431-436 (2006)

10. Bloch, S., Rosenthal, A.R., Friedman, L., Caldarolla, P.: Patient Compliance in Glaucoma. British Journal of Ophthalmology 61, 531-534 (1977) 
11. Busche, S., Gramer, E.: Improved Eyedrop Administration and Compliance in Glaucoma Patients. A Clinical Study. Klinische Monatsblatter fur Augenheilkunde Journal 211, 257-262 (1997)

12. FP7-ICT-PSP LiveCity project, http: / /www. livecity-psp.eu/

13. Patouni, E.: CIP/LiveCity - Live Video-to-Video Supporting Interactive City Infrastructure. In: The Workshop on Network Virtualization, Future Internet Assembly 2014 (FIA 2014), Athens, Greece (2014)

14. Patel, S.C., Spaeth, G.L.: Compliance in Patients prescribed Eyedrops for Glaucoma. Ophthalmic Surgery 26, 233-236 (1995)

15. Gurwitz, J.H., Glynn, R.J., Monane, M., et al.: Treatment for Glaucoma: Adherence by the Elderly. American Journal of Public Health 83, 711-716 (1993)

16. Tsai, J.C.: Medication Adherence in Glaucoma: Approaches for Optimizing Patient Compliance. Current Opinion in Ophthalmology Journal 17, 190-195 (2006)

17. Zimmerman, T.J., Zalta, A.H.: Facilitating Patient Compliance in Glaucoma Therapy. Survey of Ophthalmology Journal 28, 252-258 (1983)

18. Merentitis, A., Gazis, V., Patouni, E., et al.: Virtualization as a Driver for the Evolution of the Internet of Things: Remaining Challenges and Opportunities Towards Smart Cities. Accepted in the International Journal on Advances in Internet Technology 7(1\&2) (2014) 